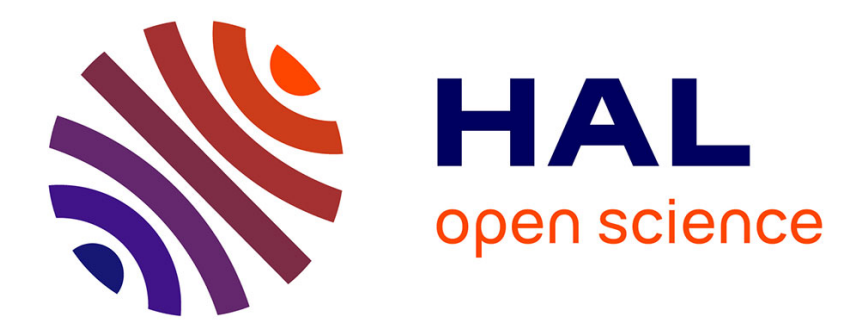

\title{
Lyapunov Stability Theory of Nonsmooth Systems
}

Daniel Shevitz, Brad Paden

\section{To cite this version:}

Daniel Shevitz, Brad Paden. Lyapunov Stability Theory of Nonsmooth Systems. IEEE Transactions on Automatic Control, 1994, 39 (9), pp.1910-1914. 10.1109/9.317122 . hal-01634266

\section{HAL Id: hal-01634266 https://hal.science/hal-01634266}

Submitted on 13 Nov 2017

HAL is a multi-disciplinary open access archive for the deposit and dissemination of scientific research documents, whether they are published or not. The documents may come from teaching and research institutions in France or abroad, or from public or private research centers.
L'archive ouverte pluridisciplinaire HAL, est destinée au dépôt et à la diffusion de documents scientifiques de niveau recherche, publiés ou non, émanant des établissements d'enseignement et de recherche français ou étrangers, des laboratoires publics ou privés. 


\section{Lyapunov Stability Theory of Nonsmooth Systems}

Daniel Shevitz and Brad Paden

\begin{abstract}
This paper develops nonsmooth Lyapunov stability theory and LaSalle's invariance principle for a class of nonsmooth Lipschitz continuous Lyapunov functions and absolutely continuous state trajectories. Computable tests based on Filipov's differential inclusion and Clarke's generalized gradient are derived. The primary use of these results is in analyzing the stability of equilibria of differential equations with discontinuous right-hand side such as in nonsmooth dynamic systems or variable structure control.
\end{abstract}

\section{INTRODUCTION}

There are many systems which have nonsmooth dynamics. Examples include systems with Coulomb friction, contact interactions, and variable structure systems where control inputs are allowed to be discontinuous. It is essential to rigorously analyze these systems and address such issues as the existence of equilibria, their stability, and qualitative dynamics. As important as nonsmooth systems are in practice, techniques are still lacking for their analysis. All classical existence theorems for ordinary differential equations require vector fields which are at least Lipschitz continuous. The aforementioned examples, and many others, fail this requirement. With respect to these classical techniques, one cannot even define a solution, much less discuss existence of equilibria and stability.

What is needed is a set of tools which allow the analysis of differential equations with discontinuous right-hand sides. The seminal contribution in this area was made by Filipov [4] who developed a solution concept for differential equations whose right-hand sides were only required to be Lebesgue measurable in the state and time variables. Using this framework, theorems were proved for existence, uniqueness, and continuous dependence on initial conditions. One area missing from this program is the stability analysis of equilibria using nonsmooth Lyapunov functions.

Yoshizawa [14] developed the Lyapunov theory for Lipschitz potential functions, but this work assumed a continuous vector field and smooth trajectories. In his book [6], Filipov studies the equilibria of differential equations with discontinuous right-hand sides, but deals with smooth Lyapunov functions.

There has been work on Lyapunov stability theory in variable structure systems; see [12], [3] for a detailed list of references. In variable structure systems one tries to pick a sliding surface and show that nearby trajectories converge first to this surface, then once on the surface, to an equilibrium point. The control is chosen in such a way that the dynamics of the closed-loop system never pass through the surface and all analysis can be done from a single side of the switching surface, and then once the trajectory is on the switching surface and smooth dynamics are restored, ordinary Lyapunov theory is adequate. As a result of this piecewise approach, the nonsmoothness of the trajectory is unimportant and differentiable Lyapunov functions suffice in many cases. For our purposes, the "kinks" are an essential part of the dynamics, and this one-sided analysis is inadequate.

The nonsmooth Lyapunov analysis of equilibria is present in the differential inclusions literature [1], [2], [7], of which Filipov's

The authors are with the Mechanical Engineering Department, University of California at Santa Barbara, Santa Barbara, CA 93111 USA. differential inclusion is a special example. Theorems are developed which rely on the notions of set valued maps and derivatives. An alternative formulation using Dini derivatives was developed by Roxin [11]. Although very general, both these techniques lack a calculus for computing derivatives of nonsmooth Lyapunov functions. We resolve this problem by exploiting the additional structure of Filipov's solution to develop more useful tools in this context. Additionally, the motivation for studying general differential inclusions is different in that the focus is on integral funnels, in which one searches for monotone trajectories which tend to the equilibrium.

The purpose of this work is to provide some tools of generalized Lyapunov analysis with which the stability properties of nonsmooth dynamic systems can be determined. These tools will turn out to be computable and easily applicable making nonsmooth Lyapunov analysis no more difficult than its smooth counterpart. Specifically, our contribution is weakening the restriction of differentiability to a broad class of nonsmooth Lipschitz continuous Lyapunov functions; the trajectories are only required to be absolutely continuous: This is important because we will show there are nonsmooth dynamic systems whose equilibria cannot be proved stable using continuously differentiable Lyapunov theory. In addition, nonsmooth Lyapunov functions are natural for nonsmooth dynamic systems.

\section{MATHEMATICAL FramewOrK}

In this section we review the Filipov solution concept for differential equations with discontinuous right-hand sides, the nonsmooth analysis of Clarke's generalized gradient, and develop a connection via a new chain rule for differentiating regular functions along Filipov solution trajectories.

\section{Filipov Solutions}

We consider the vector differential equation

$$
\dot{x}=f(x, t)
$$

where $f: R^{n} \times R \rightarrow R^{n}$ is measurable and essentially locally bounded. We must first define what it means to be a solution of this equation.

Definition 2.1 (Filipov): A vector function $x(\cdot)$ is called a solution of (1) on $\left[t_{0}, t_{1}\right]$ if $x(\cdot)$ is absolutely continuous on $\left[t_{0}, t_{1}\right]$ and for almost all $t \in\left[t_{0}, t_{1}\right]$

$$
\dot{x} \in K[f](x, t)
$$

where

$$
K[f](x, t) \equiv \bigcap_{\delta>0 \mu N=0} \overline{c o} f(B(x, \delta)-N, t)
$$

and $\bigcap_{\mu N=0}$ denotes the intersection over all sets $N$ of Lebesgue measure zero. An equivalent definition [5], [10] is: there exists $N_{f} \subset R^{m}, \mu N_{f}=0$ such that for all $N \subset R^{m}, \mu N=0$

$$
K[f](x) \equiv \overline{c o}\left\{\lim f\left(x_{i}\right) \mid x_{i} \rightarrow x, x_{i} \notin N_{f} \cup N\right\} .
$$

The content of Filipov's solution is that the tangent vector to a solution, where it exists, must lie in the convex closure of the limiting values of the vector field in progressively smaller neighborhoods around the solution point. It is important in the above definition that we discard sets of measure zero. This technical detail allows solutions to be defined at points even where the vector field itself is not defined, such as at the interface of two regions in a piecewise 


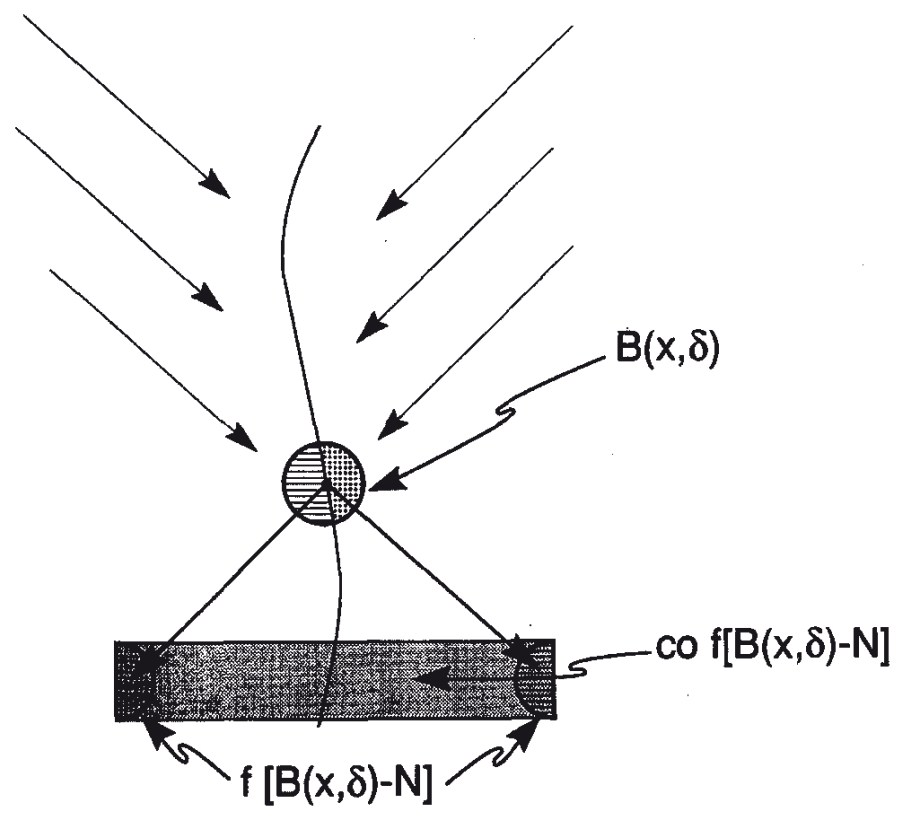

Fig. 1. The limiting procedure used to calculate $K[f](x, t)$.

defined vector field. Fig. 1 helps illustrate the limiting procedure used to define $K[f](x, t)$. This figure shows the vector images of a small neighborhood around the base point $x$. The interface is a neglected set of measure zero where the vector field is not defined. The set $K[f](x, t)$ reduces to the convex hull of two limit vectors as the neighborhood becomes vanishingly small.

Example: A nontrivial example of a nonsmooth dynamic system is the following defined for almost all $x \in R^{2}$

$$
\dot{x}=\left(\begin{array}{cc}
0 & -1 \\
1 & 0
\end{array}\right) \nabla\|x\|_{1}
$$

where $\|x\|_{1}=\left|x_{1}\right|+\left|x_{2}\right|$. The gradient in (5) is not defined on the $x_{1}$ and $x_{2}$ axes, sets of measure zero. Off the axes, the Filipov set $K[f](x)$ is a singleton

$$
K[f](x, t)= \begin{cases}\left\{(-1,+1)^{T}\right\} & \text { for } x \in \text { quadrant } 1 \\ \left\{(-1,-1)^{T}\right\} & \text { for } x \in \text { quadrant } 2 \\ \left\{(+1,-1)^{T}\right\} & \text { for } x \in \text { quadrant } 3 \\ \left\{(+1,+1)^{T}\right\} & \text { for } x \in \text { quadrant } 4 .\end{cases}
$$

On the $x_{1}$ and $x_{2}$ axes, $K[f](x, t)$ is the convex hull of each of the vectors in (6) corresponding to the quadrants which the point $x$ borders. For example, on the positive $x_{1}$ axis $K[f](x)=$ $\overline{c o}\left\{(-1,1)^{T},(1,1)^{T}\right\}$. Any Filipov solution to the differential equation (5) traces a square. observe that the trajectories in this example move along level sets of $\|x\|_{1}$, a natural (nonsmooth) Lyapunov function for this system.

In most applications the calculation of $K[f](x, t)$ involves functions $f(x, t)$ expressed as sums, products, and compositions of other functions. Hence a calculus is needed for computing the Filipov set. This calculus was derived in our earlier work [10].

We do not require Lipschitz continuity of $f(x, t)$ in $x$ or $t$ and therefore cannot in general expect uniqueness and continuous dependence on initial conditions, although we will assume both in what follows. Readers interested in more detailed discussions are referred to the original literature [4], [5].

\section{Generalized Gradients}

As shown in the example above, nonsmooth Lyapunov functions arise naturally in the stability theory of differential equations with discontinuous right-hand sides. In the application of the machinery of nonsmooth analysis, Clarke's generalized gradient [2] is particularly useful in simplifying proofs.

Definition 2.2 (Clarke's Generalized Gradient): For a locally Lipschitz function $V: R^{n} \times R \rightarrow R$ define the generalized gradient of $V$ at $(x, t)$ by

$$
\partial V(x, t)=\overline{c o}\left\{\lim \nabla V(x, t) \mid\left(x_{i}, t_{i}\right) \rightarrow(x, t),\left(x_{i}, t_{i}\right) \notin \Omega_{V}\right\}
$$

where $\Omega_{V}$ is the set of measure zero where the gradient of $V$ is not defined.

The gradient $\nabla$ includes the derivative with respect to time $(\partial / \partial t)$. In this definition, Lipschitz means Lipschitz in $(x, t)$ (discontinuities in $t$ are not allowed). For notational brevity, if a function $V(x, t)$ has no explicit $t$ dependence, we shall adopt the convention of dropping the last component of $\partial V$ which is identically zero.

One way to view the generalized gradient at a point $x$ is a set valued map equal to the convex closure of the limiting gradients near $x$. For example, the function

$$
V(x)=|x|, \quad x \in R
$$

has a generalized gradient which equals

$$
\begin{aligned}
\partial V(x) & =\{-1\} \quad x \in R^{-} \\
& =\{+1\} \quad x \in R^{+} \\
& =[-1,1] \quad x=0 .
\end{aligned}
$$

The next lemma states that the generalized directional derivative, which is defined presently, is the support function in the sense of convex analysis for the generalized gradient. Both the definition and result are due to Clarke [2].

Definition 2.3: The generalized directional derivative is defined

$$
f^{\mathrm{o}}(x ; v)=\limsup _{y \rightarrow x, t \downarrow 0} \frac{f(y+t v)-f(y)}{t} .
$$

Lemma 2.1: Let $f$ be Lipschitz near $x$, then

$$
f^{\circ}(x ; v)=\max \{\langle\xi, v\rangle \mid \xi \in \partial f(x)\} .
$$

Our chain rule will be for a useful class of functions, called regular functions [2].

Definition 2.4: $f(x, t): R^{m} \times R \rightarrow R$ is called regular if

1) for all $v$, the usual one-sided directional derivative $f^{\prime}(x ; v)$ exists,

2) for all $v, f^{\prime}(x ; v)=f^{\circ}(x ; v)$.

Examples of regular functions include smooth functions and functions which can be written as the pointwise maximum of a set of smooth functions, such as $\|x\|_{1}$. When $x(t)$ is a Filipov solution to $\dot{x}=$ $f(x, t)$ and $V(x, t)$ is a regular function, then $(d / d t) V(x(t), t)$ can be expressed in terms of $\partial V$ and $K[f](x, t)$.

Theorem 2.2 (Chain Rule): Let $x(\cdot)$ be a Filipov solution to $\dot{x}=$ $f(x, t)$ on an interval containing $t$ and $V: R^{n} \times R \rightarrow R$ be a Lipschitz and in addition, regular function. Then $V(x(t), t)$ is absolutely continuous, $(d / d t) V(x(t), t)$ exists almost everywhere and

$$
\frac{d}{d t} V(x(t), t) \epsilon^{\text {a.e. }} \dot{\dot{V}}(x, t)
$$

where

$$
\dot{\tilde{V}}(x, t):=\bigcap_{\xi \in \partial V(x(t), t)} \xi^{T}\left(\begin{array}{c}
K[f](x(t), t) \\
1
\end{array}\right) .
$$


Proof: That $V(x(t), t)$ is absolutely continuous and $(d / d t) V(x(t), t)$ exists almost everywhere is a consequence of the fact that the composition of a Lipschitz function, $V(\cdot)$, with an absolutely continuous function, $(x(t), t)$, is absolutely continuous (see, for example, [9]). At a point where $x(t)$ and $V(x(t), t)$ are both differentiable (this is true almost everywhere)

$$
\begin{aligned}
\frac{d}{d t} V(x(t), t) & \\
& =\lim _{h \downarrow 0} \frac{V(x(t+h), t+h)-V(x(t), t)}{h} \\
& =\lim _{h \downarrow 0} \frac{V(x(t)+\dot{x} h, t+h)+o(h)-V(x(t), t)}{h} \\
& =V^{\prime}\left((x, t)^{T} ;(\dot{x}, 1)^{T}\right) \\
& =V^{\circ}\left((x, t)^{T} ;(\dot{x}, 1)^{T}\right) \quad \text { by regularity } \\
& =\max \left\{\left\langle\xi \mid(\dot{x}, 1)^{T}\right\rangle \mid \xi \in \partial V(x, t)\right\} .
\end{aligned}
$$

a similar argument shows

$$
\begin{aligned}
\lim _{h \downarrow 0} & \frac{V(x(t+h), t+h)-V(x(t), t)}{h} \\
& =\min \left\{\left\langle\xi \mid(\dot{x}, 1)^{T}\right\rangle \mid \xi \in \partial V(x, t) .\right.
\end{aligned}
$$

Therefore

$$
\frac{d}{d t} V(x(t), t)=\left\langle\xi \mid(\dot{x}, 1)^{T}\right\rangle \quad \forall \xi \in \partial V(x, t) .
$$

Now, $x$ is a Filipov solution so that

$$
\dot{x}(t) \in K[f](x(t)), \quad \text { a.e. }
$$

Thus almost everywhere, $\dot{V}=\xi^{T}\left(\begin{array}{l}\eta \\ 1\end{array}\right)$ for all $\xi \in \partial V(x, t)$ and some $\eta \in K[f](x, t)$. Equivalently

$$
\dot{V} \in^{\text {a.e. }} \dot{\tilde{V}} \equiv \bigcap_{\xi \in \partial V(x(t), t)} \xi^{T}\left(\begin{array}{c}
K[f](x(t), t) \\
1
\end{array}\right) .
$$

Example: Let $V(x)=\|x\|_{1}$ and $f(x)$ as in the example, (5), above. Let $x(t)$ be the solution passing through $(1,0)^{T}$ at time $t=0$. We have

$$
\partial V(x(0), 0)=\left(\begin{array}{c}
1 \\
{[-1,+1]}
\end{array}\right)
$$

(where we have employed our convention of dropping the last term in $\partial V(x, t)$ when $V$ is independent of time) and

$$
K[f](x(0), 0)=\left(\begin{array}{c}
{[-1,+1]} \\
1
\end{array}\right) .
$$

Let $\left(1, \xi_{2}\right), \xi_{2} \in[-1,1]$ be an arbitrary element of $\partial V(x(0), 0)$ then

$$
\left[\begin{array}{l}
1 \\
\xi_{2}
\end{array}\right] K[f](x(0), 0)=\xi_{2}+[-1,+1]=\left[\xi_{2}-1, \xi_{2}+1\right]
$$

implies

$$
\dot{\tilde{V}}(x(0), 0)=\bigcap_{\xi_{2} \in[-1,1]}\left[\xi_{2}-1, \xi_{2}+1\right]=0
$$

This does not guarantee that $\dot{V}=0$ (or even exists) at this point, but $\dot{\tilde{V}}(x) \leq 0$ by which we mean $v<0$ for all $v \in \dot{\vec{V}}$, does guarantee stability. The theorems of the next section formalize this.

\section{Stability Theorems}

In this section we state two existing Lyapunov theorems (uniform stability and uniform asymptotic stability) in terms of the set valued map $\dot{\tilde{V}}$. The proofs are omitted because they are identical to their smooth counterparts except for some relations holding "almost everywhere" instead of everywhere. See Khalil and Vidyasagar [8], [13] for the smooth versions. Proofs exist for the other versions of stability, such as nonuniform, global, etc., but for the sake of brevity we have omitted them. An application of this theorem is made, and the stability of the spring-mass-coulomb friction system is proved.

Theorem 3.1: Let $\dot{x}=f(x, t)$ be essentially locally bounded and $0 \in K[f](0, t)$ in a region $Q \supset\left\{x \in R^{n} \mid\|x\|<r\right\} \times\left\{t \mid t_{0} \leq t<\right.$ $\infty\}$. Also, let $V: R^{n} \times R \rightarrow R$ be a regular function satisfying

$$
V(0, t)=0
$$

and

$$
0<V_{1}(\|x\|) \leq V(x, t) \leq V_{2}(\|x\|) \text { for } x \neq 0
$$

in $Q$ for some $V_{1}, V_{2} \in$ class $\mathcal{K}$. (See [8] for a definition of class $\mathcal{K}$ functions.) Then

1) $\dot{\tilde{V}}(x, t) \leq 0$ in $Q$ implies $x(t) \equiv 0$ is a uniformly stable solution.

2) If in addition, there exists a class $\mathcal{K}$ functions $\omega(\cdot)$ in $Q$ with the property

$$
\dot{\tilde{V}}(x, t) \leq-\omega(x)<0
$$

then the solution $x(t) \equiv 0$ is uniformly asymptotically stable.

Example ([6], [10]): Let $R(x, t)$ be a matrix which is continuous and uniformly positive definite when symmetrized and

$$
\dot{x}=-R(x(t), t) \nabla\|x\|_{1} .
$$

Then $x(t) \equiv 0$ is asymptotically stable.

Proof: Choose $V(x)=\|x\|_{1}$. Then

$$
\dot{\tilde{V}}(x, t)=\bigcap_{\xi \in \partial\|x\|_{1}} \xi^{T} K\left[-R(x, t) \nabla\|x\|_{1}\right](x, t)
$$

(since $V$ is time independent) and by the calculus for $K$ [10], we have

$$
\dot{\tilde{V}}(x, t)=\bigcap_{\xi \in \partial\|x\|_{1}}-\xi^{T} R(x, t) \partial\|x\|_{1} .
$$

Since $R$ is uniformly positive definite when symmetrized, there exists $\rho>0$ such that

$$
\xi^{T} R(x, t) \xi \geq \rho\|\xi\|^{2} .
$$

$\partial\|x\|_{1}$ is convex so

$$
\xi_{0}(x, t)=\underset{\xi \in \partial\|x\|_{1}}{\arg \min } \xi^{T}\left(\frac{R(x, t)+R^{T}(x, t)}{2}\right) \xi
$$

satisfies

$$
\begin{aligned}
\xi^{T} & \left(\frac{R(x, t)+R^{T}(x, t)}{2}\right) \xi_{0}(x, t) \\
& \geq \xi_{0}(x, t)^{T}\left(\frac{R(x, t)+R^{T}(x, t)}{2}\right) \xi_{0}(x, t) \\
& \geq \rho\left\|\xi_{0}(x, t)\right\|^{2}
\end{aligned}
$$

for all $\xi \in \partial\|x\|_{1}$. Equation (37) is a consequence of the fact that for a convex domain $D$ and a smooth function $f$ at the point $x_{0}=\arg \min f(x)$, the point $x_{0}$ satisfies

$$
\nabla f\left(x_{0}\right) \cdot\left(y-x_{0}\right) \geq 0 \text { for all } y \in D .
$$


This shows

$$
\dot{\tilde{V}}(x, t) \leq-\rho\left\|\xi_{0}(x, t)\right\|^{2} .
$$

At $x=0, \partial\|x\|_{1}=[-1,+1]^{n}$. Since $\|x\|_{1}$ is convex, $\left.\partial\|x\|_{1}\right|_{x \neq 0} \cap$ $(-1,+1)^{n}=\varnothing$ (this follows from [2, Proposition 2.2.9]). Thus

$$
\underset{\left.\xi \in \partial\|x\|_{1}\right|_{x \neq 0}}{\arg \min }\|\xi\|^{2} \geq 1
$$

so $\dot{\tilde{V}} \leq-\rho$ almost everywhere. This implies $x(t) \rightarrow 0$ asymptotically (converges in finite time in fact by the finite time stability theorem in [10]).

The proof just demonstrated shows, with minor modifications, the example in the last section where

$$
R(x, t) \equiv\left(\begin{array}{cc}
0 & -1 \\
1 & 0
\end{array}\right)
$$

has zero as a stable equilibrium point. We now argue that this equilibrium cannot be shown stable by any smooth time independent Lyapunov function.

The trajectories of this dynamic system are squares. Any nonincreasing function along a closed curve must be a constant, therefore the level sets of any proposed Lyapunov function must be squares. The level sets of any smooth function with nonzero gradients are smooth precluding the possibility of any smooth Lyapunov function for this example. A similar argument also shows the impossibility of finding a continuously differential time dependent Lyapunov function.

We now prove a nonsmooth version of LaSalle's theorem.

Theorem 3.2 (LaSalle): Let $\Omega$ be a compact set such that every Filipov solution to the autonomous system $\dot{x}=f(x), x(0)=x\left(t_{0}\right)$ starting in $\Omega$ is unique and remains in $\Omega$ for all $t \geq t_{0}$. Let $V: \Omega \rightarrow R$ be a time independent regular function such that $v \leq 0$ for all $v \in \dot{\tilde{V}}$ (if $\dot{\tilde{V}}$ is the empty set then this is trivially satisfied). Define $S=\{x \in \Omega \mid 0 \in \dot{\tilde{V}}\}$. Then every trajectory in $\Omega$ converges to the largest invariant set, $M$, in the closure of $S$.

Proof: Let $x(t)$ be a Filipov solution starting in $\Omega$. Since $V(x(t))$ is absolutely continuous, $\dot{\tilde{V}}$ is bounded below zero, and $V$ is bounded above zero, $V(x)$ tends to a constant, $a$, as $t \rightarrow \infty$. Uniqueness of trajectories implies continuous dependence on initial conditions so the positive limit set, $L^{+}$, of $x(t)$ is an invariant set. Moreover, $L^{+} \subset \Omega$ because $\Omega$ is closed. By continuity of $V$, $V(p)=a$ for all $p \in L^{+}$. Since $L^{+}$is invariant we have $\dot{V}=0$ on $L^{+}$, hence by Theorem $2.2,0 \in \dot{\tilde{V}}(x)$, a.e. in $t$. It follows by the lemma below that $L^{+} \subset \bar{S}$. Since $L^{+}$is contained in the largest invariant set in $\bar{S}$ the theorem is proved.

Lemma 3.3: Under the conditions of the theorem above

$$
L^{+} \subset \bar{S} \text {. }
$$

Proof: Let $x_{0} \in L^{+}$and $s\left(t, x_{0}, t_{0}\right)$ be the solution to $\dot{x}=f(x), x_{0}=x\left(t_{0}\right)$. Since $s\left(t, x_{0}, t_{0}\right)$ lies in $L^{+}$, $(d / d t) V\left(s\left(t, x_{0}, t_{0}\right)\right)=0$. Since $\dot{V}(x(t)) \in \dot{\tilde{V}}(x)$ a.e., in $t$, we have $s\left(t, x_{0}, t_{0}\right) \in S$ a.e. Thus $s\left(t, x_{0}, t_{0}\right) \in S$ for some $t \in\left[t_{0}, t_{0}+\delta\right)$ and all $\delta>0$. Since $s\left(t, x_{0}, t_{0}\right)$ is continuous (absolutely) we have that there are points in $S$ arbitrarily close to $x_{0}$ implying $x_{0} \in \bar{S}$. Hence $L^{+} \subset \bar{S}$.

The following theorem is a special case of a viability result in the study of differential inclusions (see [1, p. 180] for a general statement and complete proof).

Theorem 3.4: If $M$ is an invariant set in a smooth $k$-dimensional manifold $S$, then

$$
T_{m} S \cap K[f](m) \neq \varnothing
$$

for all $m \in M$.

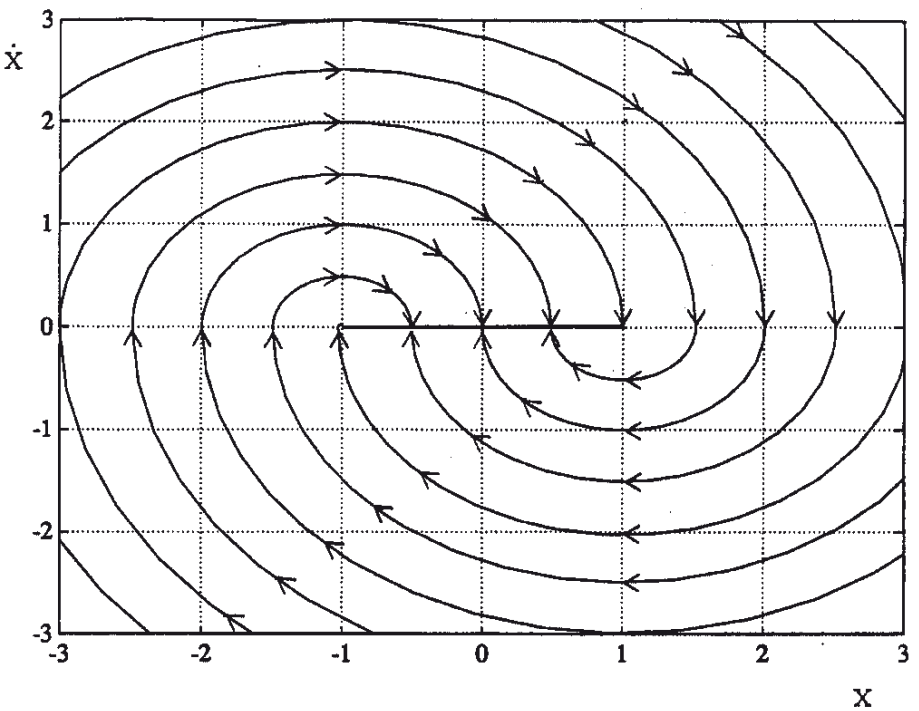

Fig. 2. The phase portrait of $m \ddot{x}+b \operatorname{sgn}(\dot{x})+k x=0$ with $m=b=k=1$.

Example: Consider a harmonic oscillator with Coulomb friction

$$
m \ddot{x}+b \operatorname{sgn}(\dot{x})+k x=0
$$

or equivalently

$$
\frac{d}{d t}\left[\begin{array}{l}
x \\
\dot{x}
\end{array}\right]=\left[\begin{array}{c}
\dot{x} \\
-\frac{b}{m} \operatorname{sgn}(\dot{x})-\frac{k}{m} x
\end{array}\right]=f(x, \dot{x}) .
$$

Choose the (smooth, time independent) Lyapunov function

$$
V(x, \dot{x})=\frac{1}{2} m \dot{x}^{2}+\frac{1}{2} k x^{2} \text {. }
$$

Then

$$
\dot{\tilde{V}}=\bigcap_{\xi \in \partial V(x, \dot{x})} \xi^{T} K\left[\begin{array}{c}
\dot{x} \\
-\frac{b}{m} \operatorname{sgn}(\dot{x})-\frac{k}{m} x
\end{array}\right] .
$$

Since $V$ is smooth

$$
\begin{aligned}
\dot{\tilde{V}} & =\nabla V^{T} K\left[\begin{array}{c}
\dot{x} \\
-\frac{b}{m} \operatorname{sgn}(\dot{x})-\frac{k}{m} x
\end{array}\right] \\
& \subset\left[\begin{array}{c}
k x \\
m \dot{x}
\end{array}\right]^{T}\left[\begin{array}{c}
\dot{x} \\
-\frac{b}{m} K[\operatorname{sgn}(\dot{x})]-\frac{k}{m} x
\end{array}\right] \\
& =-b \dot{x} \operatorname{SGN}(\dot{x}) \\
& =-b|\dot{x}|
\end{aligned}
$$

where

$$
\operatorname{SGN}(x)= \begin{cases}-1 & x<0 \\ {[-1,1]} & x=0 \\ 1 & x>0\end{cases}
$$

This implies $(x, \dot{x})$ approaches the largest invariant set in

$$
\bar{S}=\operatorname{cl}(\{(x, \dot{x}) \mid 0 \in \dot{\tilde{V}}(x, \dot{x})\}) .
$$

By Theorem 3.4

$$
T_{m} \bar{S} \cap K[f](m) \neq \varnothing
$$

for all $m \in M$. We can compute

$$
T_{m} \bar{S}=\operatorname{Span}\left[\begin{array}{l}
1 \\
0
\end{array}\right]
$$

and

$$
K[f](m)=\left[\begin{array}{c}
0 \\
-\frac{b}{m}[-1,1]-\frac{k}{m} x
\end{array}\right] .
$$

The intersection of (52) and (53) is $[0,0]^{T}$ provided $k x \in[-b, b] \mathrm{im}$ plying the largest invariant set is contained in $([-(b / k),(b / k)], 0)$. See Fig. 2 for a phase portrait. 


\section{CONCLUSION}

In this paper we have extended basic Lyapunov stability theorems to the nonsmooth case using the Filipov solution concept and Clarke's generalized gradient. The result is a theory applicable to systems with switches for which natural Lyapunov functions are often only piecewise smooth. This machinery should find application in variable structure control theory, the analysis and control of mechanical systems, and the analysis of pulse width modulated control systems.

\section{REFERENCES}

[1] Aubin and Cellina, Differential Inclusions. Berlin: Springer Verlag, 1984.

[2] F. Clarke, Optimization and Nonsmooth Analysis. New York: Wiley, 1983.

[3] R. DeCarlo, S. Zak, and G. Matthews, "Variable structure control of nonlinear multivariable systems: A tutorial," in Proc. IEEE, vol. 76, no. 3, pp. 212-232, 1988.

[4] A. F. Filipov, "Differential equations with discontinuous right-hand side," Amer. Math. Soc. Translations, vol. 42, no. 2, pp. 191-231, 1964.

[5] _ _ "Differential equations with second members discontinuous on intersecting surfaces," Differentsial'nye Uravneniya, vol. 15, no. 10, pp. 1814-1832, 1979.

[6] _. Differential Equations with Discontinuous Right Hand Sides. Boston, MA: Kluwer, 1988

[7] H. Frankowska, "Lower semicontinuous solutions of Hamilton-JacobiBellman equations," SIAM J. Contr. Optim., vol. 31, no. 1, pp. 257-272, 1993.

[8] H. Khalil, Nonlinear Systems. New York: Macmillan, 1992.

[9] I. P. Nathanson, Theory of Functions of a Real Variable. New York: Unger Publishing Co., 1961.

[10] B. Paden and S. Sastry, "A calculus for computing Filipov's differential inclusion with application to the variable structure control of robot manipulators," IEEE Trans. Circ. Syst., vol. CAS-34, no.1, pp. 73-82, 1987.

[11] E. Roxin, "Stability in general control systems," J. Differential Equations, vol. 1, pp. 115-150, 1965.

[12] V. I. Utkin, Sliding Modes and Their Applications in Variable Structure Systems. Moscow: MIR Publishers, 1978.

[13] M. Vidyasagar, Nonlinear Systems Analysis. Englewood Cliffs, NJ Prentice-Hall, 1978.

[14] Taro Yoshizawa, Stability Theory by Lyapunov's Second Method. Tokyo: Math. Soc. Japan, 1966. 